\title{
A brief review of neuropsychic symptoms associated with the female climacteric: depression and mental health
}

\begin{abstract}
Considering the tendency of improved life expectancy worldwide, women will, increasingly, experience the climacteric during the next years. A variety of biopsychosocial changes are experienced by women throughout the climacteric. As age progresses, ovarian functions decrease until the onset of menopause. With decreased levels of estradiol and progesterone, a sum of neuropsychic symptoms, such as insomnia, irritability, attention deficit and memory, anxiety and depression, become common complaints in this stage of women's life. Among them, depression is undoubtedly a global mental health problem. The aim of the present review was to present selected studies between 2007 and 2017, based on the Medline and Web of Science databases, and Academic Google, on climacteric and its main symptoms, highlighting neuropsychic symptoms, and, especially, depression. As one of the most common symptoms during climacteric, especially during menopause, depression leads to decreased motivation and productivity, increases the number of visits to health clinics, the risk of suicidal ideation and suicide itself. Studies on the prevalence and incidence of climacteric depression are of great use in the maintenance of mental health of women and in the technical qualification of outpatient clinics for women in the climacteric.
\end{abstract}

Keywords: female climacteric, aging female, menopause, neuropsychic symptoms, depression, mental health
Volume 7 Issue 4 - 2017

\author{
Cristina Wigg, 1,2,3 \\ 'Departamento de Psicometria, Universidade Federal do Rio de \\ Janeiro, Brazil \\ ${ }^{2}$ Setor de Neuropsicologia, Universidade Federal do Rio de \\ Janeiro, Brazil cristina \\ ${ }^{3}$ Coordenadora de Saúde Mental do Projeto de Criação do \\ Centro de Atenção Integral à Mulher no Climatério, do Instituto \\ Nacional de Saúde da Mulher, da Criança e do Adolescente \\ Fernandes Figueira da Fiocruz, Brazil
}

\begin{abstract}
Correspondence: Cristina Wigg; Setor de Neuropsicologia, Instituto de Neurologia Deolindo Couto, Universidade Federal do Rio de Janeiro;Av.Venceslau Brazil, 95; 2229-14 Rio de Janeiro RJ, Brazil, Email Cristina.wigg@gmail.com
\end{abstract}

Received: July 31, 2017 | Published: September 23, 2017

\section{Introduction}

Aging is a phase that is still relatively unknown compared to other life phases, although it has been increasingly studied in recent years. Female aging, characterized by entry into the climacteric, is a period comprising important hormonal, physical and neuropsychic changes. Often, neuropsychic symptoms are the first to appear, such as vasomotor disorders, headaches, anxiety, depression, fatigue, insomnia and decreased libido, among others. ${ }^{1}$

The World Health Organization (WHO) ${ }^{2}$ defines menopause as a natural and biological life phase and not a pathological process. Occurring between 45 and 55 years of age, it is characterized by a transition between the reproductive and non-reproductive period of a woman's life, as a consequence of the decrease in the production of ovarian steroid hormones, mainly estrogen and progesterone. ${ }^{2}$

A significant relationship between the presence of moderateintensity climacteric symptoms and the appearance of mood disorders has also been verified. ${ }^{3}$ Mood disorders are common complaints in climacteric women, and may be due to decreased production and catabolism of brain neurotransmitters caused by estrogen deficiency. ${ }^{1,4}$ Estrogen increases the effects of serotonin and norepinephrine, which are thought to be the neurotransmitters most related to the physiologic cause of depression. ${ }^{4}$ The relationship between depression and climacteric, and with other neuropsychic symptoms, such as heat waves, nocturnal sweating and insomnia, require further studies. Kruif et al., ${ }^{5}$ concluded in a meta-analysis that the perimenopause is a phase in which women are particularly vulnerable concerning the development of depressive symptoms, with higher symptom severity compared to the premenopause. There are indications that vasomotor symptoms are positively related to depressive symptoms during menopausal transition. According to Weber et al., ${ }^{6}$ in another metaanalysis study, the risk of depression in the perimenopause seems fairly clear, but the specific risk factors for perimenopausal depression and the trajectory over the late postmenopausal period remain to be clarified.

Other problems that frequently occur during female aging and the climacteric period may be indirectly related to biological transformations, such as the beginning of retirement, the departure of the children from home and stagnant conjugal relationships, among others, enhancing aging symptoms, leading to or potentiating depression. Thus, depression during the climacteric seems to be the result of a set of discomforts and variables, of a bio-psycho-social order, which, when present or associated, create favorable conditions for its installation or the potentiation of a disorder already present in the female subject. ${ }^{1}$

\section{Discussion}

Important changes mark the climacteric of female aging. Among a variety of symptoms, we highlight neuropsychic symptoms, which portray the turbulent and inevitable period that all women must go through. Difficulties in maintaining sleep, cognition and mood quality, among others, overlap the psychosocial experiences of maturity, requiring an absolute balance so that life can continue its course. Cognitive complaints during the climacteric usually involve deficits in attention, memory, and processing speed. Complaints regarding memory in middle age may be related to decreased attention. It has been demonstrated that premenopausal women display decreased attention, operational memory and visual abstract reasoning, while women during the peri- and postmenopausal age have shown decreased information processing speed, attention, inhibitory control and visual abstract reasoning compared to premenopausal women. ${ }^{7}$ In a cross-sectional study investigating cognition and menopause conducted with 156 women, Fernandes et al., ${ }^{8}$ concluded that the cognitive performance of menopausal women was similar to that of other sampled populations, with a greater variety of scores among individuals with lower educational levels. Thus, we may consider 
that the cognitive performance of some menopausal women may be similar to that of women who are not in the climacteric, because schooling may influence cognitive performance in general. Women with only a few years of schooling may, therefore, exhibit worse cognitive performance regardless of age and whether or not they are in the climacteric.

Regarding cognitive and affective changes during the climacteric, Weber's et al. ${ }^{6}$ meta-analysis study argued that there are still many questions about this topic. The authors cite, for example, that many studies have not considered the hormonal differences between the early and late perimenopause and postmenopausal stages, and that there is evidence to suggest that there are important differences between these stages. According to the authors, several studies reviewed only a single stage of perimenopause or postmenopausal. This is a very important methodological issue, which suggests that further studies should consider such differences.

In addition to cognitive difficulties, sleep disorders during the climacteric are also quite common, and their relationship with other variables such as depression, anxiety and psychosocial aspects is quite relevant. ${ }^{1,9,10}$ The study by Machado et al., ${ }^{11}$ evaluated factors associated with hypertension, diabetes, insomnia and depression in Brazilian women between 40 and 65, with 11 years or more of schooling. The authors concluded that insomnia was the most prevalent morbidity, followed by depression, hypertension and diabetes. According to Makara-Studzińśka et al., ${ }^{12}$ in an intercontinental review study the most common, troublesome symptoms of menopause age include depressive disorders, sleep disorders, sexual dysfunction, discomfort associated with muscle pain, joint aches, osteoporosis and characteristic hot flashes. Polo-Kantola ${ }^{9}$ observed that sleep problems in middle-aged women were related to mental and mood disorders, as well as decreased estrogen and progesterone levels. According to the author, in addition to decreased sleep quality, specific sleep disorders, such as sleep-disordered breathing and restless-leg syndrome, become more prevalent during middle age and, especially, after menopause. The qualitative study performed by Vigeta et al., ${ }^{10}$ conducted with postmenopausal women found that psychosocial factors were associated with insomnia, and Doo \& $\mathrm{Kim}^{13}$ verified that short sleep duration was associated with increased levels of obesityrelated variables and increased consumption of $\mathrm{CHO}$-rich foods in premenopausal women. Sleep deteriorates with age, and complaints of insomnia increase significantly from menopause. Insomnia can occur as a secondary disorder for heat waves, mood disorders, medical conditions, psychosocial factors, underlying intrinsic sleep disorders such as obstructive sleep apnea (OSA) or restless leg syndrome (RLS), or it can be a primary disorder. ${ }^{14}$

Despite the importance of adequate cognitive functioning and sleep and maintaining weight for physical and mental well-being, depression is one of the most troubling neuropsychological symptoms associated with the climacteric, from a point of view of maintaining physical and mental health. Depression, its different and varied causes, and its effects on health and the maintenance of quality of life has been much studied, and represents an important field of investigation in mental health. According to the $\mathrm{WHO}^{15}$ depression is the 4th world cause of social incapacitation and the main public health problem worldwide, with an estimated prevalence of depressive disorders among the elderly being between 10 and $20 \%$, depending on the cultural situations. In an prospective, analytic study of 70 climacteric women, Silva et al., ${ }^{16}$ verified a high prevalence of depression in the evaluated patients, observing a percentage of $34.3 \%$ of depression, and $70.8 \%$ were classified as mild intensity according to Hamilton scale. According to the authors, an association was found between the presence of depression and patients with vasomotor symptoms (p $=0.03)$, insomnia $(\mathrm{p}<0.001)$, menopause $(\mathrm{p}=0.05)$, with a history of post-partum depression $(\mathrm{p}=0.04)$ and premenstrual dysphoric disorder $(\mathrm{p}=0.05)$ and negative menopausal vision $(\mathrm{p}=0.001)$. Yangin et al., ${ }^{17}$ in a study conducted with 300 menopausal women, found that $29.3 \%$ of women displayed depression and $65 \%$ presented sexual dysfunctions. The prevalence of depression and anxiety in a total of 1280 women between the ages of 45 and 59 were 306 $(23.9 \%)$ and $131(10.2 \%)$, respectively. ${ }^{18}$ In a recent study, Bener et al., ${ }^{19}$ evaluated the presence of and association between depression, stress and anxiety in 1468 women between the ages of 45 and 65 . The highest prevalence was for depression (31.2\%), compared to $22.9 \%$ for stress and $14.1 \%$ for anxiety. Polisseni et al., ${ }^{3}$ found that depression was more frequent in women with anxiety and insomnia. According to the authors, there is a significant relationship between the presence of climacteric symptoms of moderate intensity and the appearance of mood swings.

In an important study on depression and menopause, Soares ${ }^{20}$ reported that depression is a disabling condition, which often leads to significant personal, social and economic costs. In addition, the author noted that depression in women is probably associated with increased sensitivity of some women to changes in the hormonal environment that occur during the luteal phase of their cycles, during the postpartum period and/or during the menopause transition. According to the author, there is controversy surrounding menopausal depression, due to the lack of methodological rigor to characterize reproductive staging or to evaluate psychiatric conditions during middle age.

The results of a recent meta-analysis ${ }^{21}$ indicated the involvement of estrogen deficiency in the pathophysiology of late depression, which could allow the identification of a group of women at greater risk of depression that could benefit from therapies based on estrogen and psychiatric monitoring.

A recent study on quality of life and the climacteric involving 100 peri- and postmenopausal women between 40 and 60 years old, found that the occurrence of vasomotor symptoms was average, while the most prevalent psychosocial symptoms reported were feelings of anxiety and nervousness (94\%) and overall depression (88\%). ${ }^{22}$ The physical symptoms were quite variable in occurrence, such as fatigue, decreased physical strength and lack of energy in $93 \%$ of the evaluated women. The study concluded that menopause does indeed cause physical and psychic problems, and that important interventions and appropriate educational strategies are required for a better quality of life among climacteric women. ${ }^{22}$

Soares $^{23}$ reported that the presence of vasomotor symptoms (hot flashes) interfering with sleep, can determine the appearance of depressive and anxious conditions (Domino Theory) in the long term. However, Greendale et al., ${ }^{24}$ found no relation between vasomotor symptoms and cognitive performance, contrary to the "cascade theory", in which vasomotor symptoms lead to disturbed sleep, fatigue, depressive symptoms, and memory problems.

A community-based sample of 190 middle-aged women was studied to examine the impact of the previous diagnoses of depression, menopause status, vasomotor symptoms, and neuroticism on depressive symptoms among menopausal women in Taiwan over a 30 -month follow-up. ${ }^{25}$ The results of the hierarchical multiple regression analyses showed that depressive symptoms during the menopause transition predicted depressive symptoms over 30months. After controlling for depressive symptoms during the menopause transition, the previous diagnoses of depression, menopause status, and vasomotor symptoms could not predict depressive symptoms over 
30months, whereas neuroticism still predicted depressive symptoms over 30 months. The authors suggested that neuroticism plays an important role in the persistence of depression among climacteric women after 30 months.

In addition to worsening other climacteric symptoms, depression has shown strong associations with suicidal ideation. Statistics show that women are 4 times more likely to have suicidal ideations than men. Postmenopausal women may have a higher suicidal ideation than premenopausal women, ${ }^{26,27}$ and postmenopausal women undergoing hormone replacement therapy (HRT), especially for more than 10 years, have shown a higher suicidal ideation compared to postmenopausal women not undergoing TRH. ${ }^{27}$ Physicians should pay attention to the symptoms of mood and suicidal ideation in postmenopausal women with HRT. $^{27}$ The relationship between suicidal ideation and climacteric is still not entirely clear, considering the variety of biopsychosocial factors and conditions that could spark suicidal thoughts. However, although we can not state that lowering estrogen levels leads to depression, we must consider that metabolic and hormonal factors may be associated with increased mood regulation, leading to increased risk for depression during menopause. ${ }^{28}$ Thus, it is a fact that depression often accompanies the stages of the climacteric and therefore, mental health professionals must be aware of the hormonal, physical, emotional and psychosocial changes in women during the climacteric. A climacteric woman with a complaint of depression must be properly investigated and assisted, at the risk of developing suicidal thoughts and attempts. Preventive methods, undoubtedly, are still our greatest allies in controlling depression and suicide.

\section{Conclusion}

As seen herein, changes occur during the period of female climacteric and a variety of physical and neuropsychic symptoms have important implications on women's mental health. Of the many neuropsychic symptoms identified during the climacteric, depression shows a high prevalence, and associations with other symptoms are almost always present, such as sleep, cognition, overweight, stress and anxiety, among others. Being a pre-existing symptom, potentiated by the climacteric, and/or due to the physical and/or psychological changes implied in the aging itself, depression is undoubtedly a serious problem for climacteric women's health services, which attend a number of depressed, unproductive and unhappy women.

We can understand how difficult it is to live and maintain control of all the symptoms caused by the climacteric. Studies with high descriptive potential, aiding in identifying and treating depression, insomnia and other climacteric neuropsychic symptoms, are increasingly necessary, given the increases in life expectancy. In addition, depression and its limitations and risks to women's physical and mental health represent an increasingly present problem in families worldwide and therefore, new and more effective assistance policies to climacteric women must to be considered, in order to produce actions that actually rehabilitate women during their aging process.

So many physical and psychic changes identified during the climacteric, combined with social changes, represent a sum of conditions that demand special attention to the diagnosis and intervention in ambulatory care for the climacteric woman. Considering the most frequently mentioned neuropsychic symptoms, the diagnostic procedure should include examinations on cognitive and behavioral profiles, including depression, anxiety, insomnia and stress evaluations. The interventions must be educational, preventive and bio-psycho-social. Ambulatory care should be humanized, valuing individual differences, considering the variety of symptoms found in the female climacteric and the peculiarities of each woman. Monitoring and controlling depression should be a priority, considering its implications and the risk of complications over other symptoms.

\section{Conflicts of interest}

There is no conflict of interest to declare.

\section{Acknowledgments}

None.

\section{Funding}

None.

\section{References}

1. Ministério da Saúde. Secretaria de Atenção à Saúde. Departamento de Ações Programáticas Estratégicas. Manual de Atenção à Mulher no Climatério/Menopausa. (MS), Brasil. 2008.

2. Organização Mundial da Saúde. Investigaciones sobre la menopausia em los años noventa. Ginebra. Informe de um Grupo Cient'ifico de la OMS. Serie de Informes Técnicos. 1996;866.

3. Polisseni AF, Araújo DACD, Polisseni F, et al. Depressão e ansiedade em mulheres climatéricas: fatores associados. Rev Bras Ginecol Obstet. 2009;31(1):28-34.

4. Dalal PK, Agarwal M. Postmenopausal syndrome. Indian J Psychiatry. 2015;57(Suppl 2):222-232.

5. de Kruif M, Spijker AT, Molendijk ML. Depression during the perimenopause: A meta-analysis. J Affect Disord. 2016;206:174-180.

6. Weber MT, Maki PM, McDermott MP. Cognition and mood in perimenopause: a systematic review and meta-analysis. J Steroid Biochem Mol Biol. 2014;142:90-98.

7. Santiago VLG. Cognição no climatério em mulheres com migrânea 2012.

8. Fernandes RDCL, Silva KSD, Bonan C, et al. Avaliação da cognição de mulheres no climatério com o Mini-Exame do Estado Mental e o Teste de Memória da Lista de Palavras, Cadernos de Saúde Pública. 2009.

9. Polo-Kantola P. Sleep problems in midlife and beyond. Maturitas. 2011;68(3):224-232.

10. Vigeta SM, Hachul H, Tufik S, et al. Sleep in postmenopausal women. Qual Health Res. 2012; 22(4):466-475.

11. Machado VDSS, Valadares ALR, Costa-Paiva L, et al. Morbidity and associated factors in climacteric women: a population based study in women with 11 or more years of formal education. Rev Bras de Ginec e Obst. 2012;34(5):215-220

12. Makara-Studzińśka MT, Kryś-Noszczyk KM, Jakiel G. Epidemiology of the symptoms of menopause-an intercontinental review. Prz Menopauzalny. 2014;13(3):203-211.

13. Doo M, Kim Y. The risk of being obese according to short sleep duration is modulated after menopause in Korean women. Nutrients. 2007;9(3):206-217.

14. Bruyneel M. Sleep disturbances in menopausal women: Aetiology and practical aspects. Maturitas. 2015;81(3):406-409.

15. Organização Mundial da Saúde. Saúde mental: nova concepção, nova esperança. Ginebra. 2001.

16. Silva, MNMD, Brito LMO, et al. Depressão em mulheres climatéricas: análise de mulheres atendidas ambulatorialmente em um hospital universitário no Maranhão. Revista de Psiq RG Sul. 2008;30(2):150-154 
17. Yangın HB, Sözer GA, Şengün N,et al. The relationship between depression and sexual function in menopause period. Maturitas. 2008;61(3):233-237.

18. Li Y, Yu Q, Ma L, et al. Prevalence of depression and anxiety symptoms and their influence factors during menopausal transition and postmenopause in Beijing city. Maturitas. 2008;61(3):238-242.

19. Bener A, Saleh NM, Bakir A, et al. Depression, Anxiety, and Stress Symptoms in Menopausal Arab Women: Shedding More Light on a Complex Relationship. Ann Med Health Sci Res. 2017;6(4):224-231.

20. Soares CN. Depression and Menopause: Current Knowledge and Clinical Recommendations for a Critical Window. Psychiatr Clin North Am. 2017;40(2):239-254.

21. Georgakis MK, Thomopoulos TP, Diamantaras AA, et al. Association of age at menopause and duration of reproductive period with depression after menopause: a systematic review and meta-analysis. JAMA psychiatry. 2016;73(2):139-149.

22. Karmakar N, Majumdar S, Dasgupta A, et al. Quality of life among menopausal women: A community-based study in a rural area of West Bengal. J Mid-life Health. 2017;8(1):21-27.
23. Soares CN. Depression during the menopausal transition: window of vulnerability or continuum of risk? Menopause. 2008;15(2):207-209.

24. Greendale GA, Wight RG, Huang MH, et al. Menopause-associated symptoms and cognitive performance: results from the study of women's health across the nation. Am J Epidemiol. 2010; 171(11):1214-1224.

25. Chou CH, Ko HC, Wu JYW, et al. Effect of previous diagnoses of depression, menopause status, vasomotor symptoms, and neuroticism on depressive symptoms among climacteric women: A 30-month followup. Taiwan J Obstet Gynecol. 2015;54(4):385-389.

26. Kornstein SG, Young EA, Harvey AT, et al. The influence of menopausal status and postmenopausal use of hormone therapy on presentation of major depression in women. Menopause. 2010;17(4):828-839.

27. Lee JY, Park YK, Cho KH, et al. Suicidal ideation among postmenopausal women on hormone replacement therapy: the Korean National Health and Nutrition Examination Survey (KNHANES V) from 2010 to 2012. $J$ Affect Disord. 2016;189:214-219.

28. Berent-Spillson A, Marsh C, Persad C, et al. Metabolic and hormone influences on emotion processing during menopause. Psychoneuroendocrinology. 2017;76:218-225. 